\title{
Prevalence and Comparison of Visual Acuity Charts in School Going Children with Low Vision in Garhwal Himalayan Region
}

\author{
Achyut Pandey* \\ Department of Ophthalmology, VCSGMC\&RI, India
}

Submission: August 27, 2015; Published: September 05, 2015

*Corresponding author: A N Pandey, Room no 61, Dept of Ophthalmology, VCSGMC\&RI, Srinagar garhwal, Dist pauri garhwal, Uttarakhand-246174, email: achyutpandey@gmail.com

\begin{abstract}
Aim: To compare the effectiveness of visual acuity charts used in school going children with Low Vision.

Materials and Methods: Two groups of 40 each of the children age less than 15 years of age, 40 each in study and control group attending the out patients department were selected after fulfilling the inclusion criteria. All the children were tested with Snellen's and Lea's symbol chart. The response of each child with both type charts was recorded. Testing of both groups was done with and without correction.

Results: In groups, female preponderance, 24(60\%) in study group, 22(55\%) were female children in control group. Majority of children in study group had refractive error (40\%) and amblyopia (37.5\%) while in control group 60\% of children were found to have myopia. In control group, before correction, visual acuity was more than 6/18 in 13 (32.5\%) children in Snellen's chart and 22 (55\%) children in Lea's chart while 27 (67.5\%) children were less than 6/18 in Snellen's chart, 18 (45\%) children in Lea's chart. In study group, before correction visual acuity was less than 6/18 in 37 (93.2\%) in Snellen's chart and 32 (79.4\%) children in Lea's chart while after correction the visual acuity was more than 6/18 in (17) 41.3\% of cases in Snellen's chart and (30) 75.8\% of cases in Lea's chart.
\end{abstract}

Conclusion: Visual performance depends not only on size and distance, but also on contrast, illumination, and arrangement of targets. Study also concluded myopia and astigmatism is the commonest refractive error among school going children.

\section{Introduction}

Vision or visual perception is the complex integration of light sense, form sense, contrast sense and colour sense. Visual acuity measurement involves the determination of a threshold level of vision. Visual acuity is represented as the reciprocal of the minimal angle of resolution (the smallest letters resolved) at a given distance at high contrast. Visual acuity testing especially in low vision depends on targets, distance, lighting, background, contrast, ocular condition, concentration and intellectual level of the patients. Selection of a test is dependent on ability to respond, developmental age and time of testing. Type of tests used, therefore, will modify responses elicited. This is true in children especially in those with low vision [1]. Different types of visual acuity charts which are using now a days to Snellens acuity chart, Lea symbols test, Bailey lowie chart, ferris Log MAR charts, Wateloo charts, HOTV tests, Landolts test types and Lighthouse flash card test.

\section{Aim}

To compare the effectiveness of visual acuity charts used in school going children with Low Vision.

\section{Materials and Methods}

This is the prospective and comparative non randomized study done in outpatient department of a tertiary eye care centre over a period of 1 year. Two groups of 40 each of the children attending the Pediatric outpatients department were selected. 40 were included as control group of the children with low vision while 40 were included in the study group. All the children in both the groups were tested with Snellen's chart and Lea's symbol chart. Chart used was according to their developmental age. Those in the low vision group were tested at different distances also. The response of each child with both type charts was recorded. Testing of both groups was done by the same person and both groups were tested with and without correction.

\section{Inclusion criteria}

Both sexes

Children with age less than 15 years 
All normal and refractive error children

Children with low vision

\section{Exclusion criteria}

Children above 16 years

Children with multi handicap

Infants.

\section{Basic Protocol}

A standard protocol was used to collect and document all the details regarding the cases included in this study. Detailed information about the history and complaints of the patients were taken. This included the type of visual problems, duration of symptoms and any history of predisposing factors like refractive errors, family history, systemic disease etc.,

\section{Data collection}

Data includes demographic details of children (age, sex,), type of refractive error, and duration of symptoms, prior history of using glasses, surgery and visual acuity at presentation were collected. Complete ocular examination details of each patient such as visual acuity for distant vision (checked with Snellen's acuity chart and Lea's symbol chart), Slit lamp examination, fundus examination (done with +90D lens) and binocular indirect ophthalmoscopy, were recorded. Data regarding subjective correction were also noted. Visual acuity was measured before and after correction with Snellen's chart and Lea's symbol chart was also noted.

\section{Procedure of testing in Snellen's chart}

For testing distant visual acuity, the patient was seated at a distance of $6 \mathrm{~m}$ from the Snellen's chart, so that the rays of light are practically parallel and the patient exerts minimal accommodation. The charts were properly illuminated not less than 20 foot-candles. The patient was asked to read the chart with each eye separately and the visual acuity was recorded as a frication, the numerator being the distance of the patient from the letters and the denominator being the smallest letters accurately read. When the patient was able to read up to $6 \mathrm{~m}$ the visual acuity was recorded as $6 / 6$, which is normal. Similarly, depending upon the smallest line that the patient can read from the distance of $6 \mathrm{~m}$, his or her vision was recorded as $6 / 9,6 / 12$, $6 / 18,6 / 24,6 / 36,6 / 60$. If one cannot see the top line from $6 \mathrm{~m}$, he or she was asked to slowly walk towards the chart till one can read the top line. Depending upon the distance at which one can read the top line; the vision was record as $5 / 60,4 / 60,3 / 60,2 / 60$, $1 / 60$.

If the patient was unable to read the top line even from $1 \mathrm{~m}$, he or she was asked to count fingers of the examiner. His or her vision was recorded as CF-3, CF-2, CF-1, or CF close to face, depending upon the distance at which the patient was able to count fingers. When the patient failed to count fingers, the examiner moved his or her hand close to the patient's face. If one can appreciate the hand movements, the examiner noted whether the patient can perceive light or not, if yes, vision was recorded as projection of light positive and, if not, it was recorded as projection of light negative.

\section{Procedure of testing in Lea's chart}

The child was allowed to stand or sit at a table with the response card in front, eyes at a 10-foot distance from the chart. The child must be conditioned to match symbols by pointing to the same symbol on the response card as was being shown with a flash card or pointed to on the chart. Begin screening with one person holding an occluder over the child's left eye, another person pointed to the symbols on the Lea wall chart. The screener pointed to the symbols must be careful not to cover the box around the symbols as this could affect the results of the test. The child should point to the corresponding symbol on the response card. Start with the top line of the chart and continue downward showing one letter per line. If the child reaches the bottom line, show the remaining three symbols. If the child misses any of the symbols, go to the line above and show four different symbols in that line. If the child matches them correctly, proceed downward. To receive credit for a line, the child must correctly match each of the four different symbols on the line. The number recorded as the visual acuity is the smallest line the child can read correctly. The procedure is repeated for the left eye.

\section{Results}

This prospective and comparative study was conducted at the tertiary eye care hospital over a period of one year between May 2012 to April 2013. 160 eyes of 80 patients with refractive errors were included in this study.

\section{Age distribution}

In control group (normal children), 6 of 40 (15\%) were $0-5$ age group, and $9(22.5 \%)$ children were 6-10 age group and 25 (62.5\%) children were 11-15 age group (Table 1).

In study group (low vision children), 4 of $40,(10 \%)$ children were 0 -5 age group and 9 (22.5\%) children were 6-10 age group and $27(67.5 \%)$ children were $11-15$ age group (Table 1$)$.

\section{Sex distribution}

In control group, 16 of $40(40 \%)$ were male children and $24(60 \%)$ were female children (Table 2).

In study group, 18 of $40(45 \%)$ were male children and $22(55 \%)$ were female children (Table 2).

\section{Diagnosis of low vision}

In study group, 16 of 40 (40\%) had refractive errors and 15 $(37.5 \%)$ children had amblyopia and $5(12.5 \%)$ children had corneal disorders and 2 (5\%) children had glaucoma (Table 3).

\begin{tabular}{|c|c|c|c|c|}
\hline \multicolumn{2}{|c|}{ Table 1: Age distribution. } \\
\hline \multirow{2}{*}{ Age } & \multicolumn{2}{|c|}{ Control group } & \multicolumn{2}{|c|}{ Study group } \\
\cline { 2 - 5 } & No. patients & $\%$ & No. patients & $\%$ \\
\hline $0-5$ & 6 & 15 & 4 & 10 \\
\hline $6-10$ & 9 & 22.5 & 9 & 22.5 \\
\hline $11-15$ & 25 & 62.5 & 27 & 67.5 \\
\hline
\end{tabular}


Table 2: Sex distribution.

\begin{tabular}{|c|c|c|c|c|}
\hline \multirow{2}{*}{ Sex } & \multicolumn{2}{|c|}{ Control group } & \multicolumn{2}{c|}{ Study group } \\
\cline { 2 - 5 } & No. patients & $\mathbf{\%}$ & No. patients & $\%$ \\
\hline Male & 16 & 40 & 18 & 45 \\
\hline Female & 24 & 60 & 22 & 55 \\
\hline
\end{tabular}

\section{Type of refractive errors}

In control group, of the 40 children, 24 were found to be myopia (60\%), hypermetropia in $2(5 \%)$ and astigmatism in 14 (35\%) children.

In study group, of the 40 children, it was found that myopia in $24(60 \%)$, hypermetropia in $10(25 \%)$ and astigmatism in 6 (15\%) children. (Table 4).

\section{Visual acuity}

\section{Control group:}

a) Before correction: In the control group, of 40 children, visual acuity was more than 6/18 in $13(32.5 \%)$ children in Snellen's chart and 22 (55\%) children in Lea's chart. 27 (67.5\%) children were less than 6/18 in Snellen's chart, 18 (45\%) children in Lea's chart (Table 5).

b) After correction: In the control group of 40 children had visual acuity was more than $6 / 18$ in $40(100 \%)$ children in both Snellen's and Lea's chart (Table 5). There was different in visual

\begin{tabular}{|c|c|c|}
\hline \multicolumn{2}{|l|}{ Table 3: Diagnosis of low vision. } \\
\hline Diagnosis & No. of children & $\%$ \\
\hline Refraction & $\mathbf{1 6}$ & $\mathbf{3 9}$ \\
\hline Amblyopia & $\mathbf{1 5}$ & $\mathbf{3 8}$ \\
\hline Neuro & $\mathbf{5}$ & $\mathbf{1 3}$ \\
\hline Cornea & $\mathbf{2}$ & $\mathbf{5}$ \\
\hline Glaucoma & 2 & 5 \\
\hline
\end{tabular}

Table 4: Type of refractive errors.

\begin{tabular}{|c|c|c|c|c|}
\hline $\begin{array}{c}\text { Types of refractive } \\
\text { error }\end{array}$ & $\begin{array}{c}\text { Study } \\
\text { Group }\end{array}$ & $\mathbf{\%}$ & Control group & $\mathbf{\%}$ \\
\hline Myopia & 24 & 60 & 24 & 60 \\
\hline Hypermetropia & 10 & 25 & 2 & 5 \\
\hline Astigmatism & 6 & 15 & 14 & 35 \\
\hline
\end{tabular}

Table 5: Control group visual acuity.

\begin{tabular}{|c|c|c|c|c|}
\hline \multicolumn{5}{|c|}{ Before correction } \\
\hline VA & Snellen's & \% & Lea's & $\%$ \\
\hline$>6 / 18$ & 13 & 32.5 & 8 & 55 \\
\hline$<6 / 18$ & 27 & 67.5 & 32 & 45 \\
\hline \multicolumn{5}{|c|}{ After correction } \\
\hline VA & Snellen's & $\%$ & Lea's & $\%$ \\
\hline$>6 / 18$ & 40 & 100 & 40 & 100 \\
\hline$<6 / 18$ & 0 & 0 & 0 & 0 \\
\hline
\end{tabular}

acuity with different charts in $22.5 \%$ of cases before correction. Visual acuity did not differ between charts, in all cases after correction.

\section{Study group:}

a) Before correction: In the study group, out of 40 cases, the visual acuity was more than $6 / 18$ in $3(6.8 \%)$ children in Snellen's chart, $8(20.6 \%)$ children in Lea's chart. Visual acuity was less than 6/18 in 37 (93.2\%) children in Snellen's chart and $32(79.4 \%)$ children in Lea's chart (Table 6).

b) After correction: In the study group, out of 40 children, the visual acuity was more than $6 / 18$ in (17) $41.3 \%$ of cases in Snellen's chart and (30) $75.8 \%$ of cases in Lea's chart. Visual acuity was less than 6/18 in $23(58.7 \%)$ children in Snellen's chart and 10 (24.2\%) children in Lea's chart (Table 6).

\section{Discussion}

This prospective and comparative study was conducted in the tertiary eye care hospital from May 2014 to February 2015. 160 eyes of 80 patients with refractive errors were included in this study. Niroula et al [2] reported that distribution of myopia was found to be higher $(4.05 \%)$ than the hyperopia $(1.24 \%)$ and astigmatism (1.14\%). Niroula et al [2] in the present study, myopia was in $24(60 \%)$, hypermetropia in $2(5 \%)$ and astigmatism in $14(35 \%)$ children. In study group out of 40 children we found to be myopia in $24(60 \%)$, hypermetropia in $10(25 \%)$ and astigmatism in $6(15 \%)$ children. Tong et al reported bearing in mind that the visual acuity measurements were performed by two different groups of professionals, visual acuity screening using the ETDRS method appears to be more accurate than the simplified charts for the detection of myopia or any refractive errors in children. Tong et al [3] In present study Lea's symbol chart was found to be better than Snellen's chart by measuring the accurate visual acuity among the normal as well as the low vision children. Dobson et al [4] reported correlation between visual acuity results obtained with the two charts was high. There was no difference in absolute inter-eye acuity difference measured with the two acuity charts. However, on average, Lea's Symbols acuity scores were one log MAR line better than Bailey-Lovie acuity scores, and this difference increased with worse visual acuity. Cyert L et al [5], Lueder G [6] In present study also there was no much difference in normal children comparatively than low vision children.

Tong et al [7] studied modified ETDRS visual acuity chart can

Table 6: Study group visual acuity.

\begin{tabular}{|c|c|c|c|c|}
\hline \multicolumn{5}{|c|}{ Before correction } \\
\hline VA & Snellen's & \% & Lea's & $\%$ \\
\hline$>6 / 18$ & 3 & 6.8 & 8 & 20.6 \\
\hline$<6 / 18$ & 37 & 93.2 & 32 & 79.4 \\
\hline \multicolumn{4}{|c|}{ After correction } \\
\hline VA & Snellen's & $\%$ & Lea's & $\%$ \\
\hline$>6 / 18$ & 17 & 41.3 & 30 & 75.8 \\
\hline$<6 / 18$ & 23 & 58.7 & 10 & 24.2 \\
\hline
\end{tabular}


be used to predict refractive errors in school children in Singapore in a sensitive and specific manner using a referral criterion of worse than or equal to 0.28 logarithm of the minimum angle of resolution. Dobson et al [8] reported in the comparitive visual acuity study of young children, in whom the primary source of reduced visual acuity was astigmatism-related amblyopia, the Lea's symbols chart produced visual acuity scores that were about 0.5 lines better than visual acuity scores obtained with ETDRS charts. Dobson et al [8] Similarly, in the present study also Lea's symbol visual chart produced visual acuity scores better than the Snellen's chart especially in low vision children. Uzma et al [9] found out that myopia was present in almost $51 \%$ of the study population, she stressed on provision of health education, periodic visual screening programs, and primary eye care by trained health care personnel in the elementary schools will prevent the prevalence of refractive errors and common ocular diseases in school children. Gupta et al [10] found out that refractive errors were the most common ocular disorders among school children of age 6-16 years.

Dandona et al [11] in his study stated that the prevalence of uncorrected refractive error, especially myopia, was higher in urban children. He further emphasized on the eye screening of school children. However, the approach used may be different for urban and rural school children [11,12].

\section{Conclusion}

Visual performance depends not only on size and distance, but also on contrast, illumination, and arrangement of targets. These factors, affected in low vision, may modify responses during testing, thus giving high false negatives. Use of appropriate charts is, therefore, mandatory while assessing children with low vision. This study also concluded myopia and astigmatism is the commonest refractive error among school going children Lea's chart is easier to measure visual acuity in low vision school going children than the Snellen's chart. It was observed that the visual acuity differed with different charts, in low vision cases, before and after correction. The difference is dependent on the individual's absolute level of visual acuity. Visual acuity is easy to measure in children by Lea's chart.

\section{Acknowledgement}

The authors are thankful to Dr. Amjad Salman, Dr. Preeti Pant and Divyam Pandey for their timely help.

\section{References}

1. Khurana AK (2008) Theory and Practice of Optic and Refraction. 2nd Edition, 39-45.

2. Niroula DR, Saha CG (2004) Study on the refractive errors of school going children of Pokhara city in Nepal. Kathmandu Univ Med J 7(25): 67-72.

3. Tong L, Saw SM, Chan ES, Yap M, Lee HY (2004) Screening for myopia and refractive errors using Log MAR visual acuity by optometrists and a simplified visual acuity chart by nurses. Optom Vis Sci 81(9): 684691.

4. Dobson V, Maguire M, Orel-Bixler D, Quinn G, Ying GS, et al. (2003) Vision in Preschoolers (VIP) Study Group, Visual acuity results in school-aged children and adults: Lea Symbols chart versus BaileyLovie chart. Optom Vis Sci 80(9): 650-654.

5. Cyert L, Schmidt P, Maguire M, Moore B, Dobson V, et al. (2003) Threshold visual acuity testing of preschool children using the crowded HOTV and Lea Symbols acuity tests. J AAPOS 7(6): 396-399.

6. Lueder G, Garibaldi D (1997) Comparison of visual acuity measured with Allen figures and Snellen letters using the B-VAT II monitor. Ophthalmology 104(11): 1758-1761.

7. Tong L, Saw SM, Tan D, Chia KS, Chan WY, et al. (2002) Sensitivity and specificity of visual acuity screening for refractive errors in school children. Optom Vis Sci 79(10): 650-657.

8. Dobson V, Clifford-Donaldson CE, Miller JM, Garvey KA, Harvey EM. (2009) A comparison of Lea Symbol vs ETDRS letter distance visual acuity in a population of young children with a high prevalence of astigmatism. JAAPOS 13(3): 253-257.

9. Nazia Uzma, Santosh Kumar, Moinuddin salar, Devender Reddy (2009) A comparative clinical survey of the prevalence of refractive errors and eye diseases in urban and rural school children. Can J Ophthalmol 44(3): 328-333.

10. Madhu Gupta, Bhupinder Gupta, Anil Chauhan, Ashok Bharadwaj (2009) Ocular morbidity prevalence among school children in Shimla, Himachal, North India. Indian J Ophthalmol. 57(2): 133-138.

11. Dandona R, Dandona L, Srinivas M, Sahare P, Narsaiah S, et al. (2002) Refractive error in children in a rural population in India. Invest Ophthalmol Vis Sci 43(3): 615-622.

12. Dandona R, Dandona L Srinivas M, Giridhar P, McCarty CA, et al. (2002) Population-based assessment of refractive error in India: the Andhra Pradesh eye disease study. Clin Experiment Ophthalmol 30(2): 84-93. 